\title{
Approximation by Parametric Extension of Szász-Mirakjan- Kantorovich Operators Involving the Appell Polynomials
}

\author{
Md. Nasiruzzaman (i) and A. F. Aljohani \\ Department of Mathematics, Faculty of Science, University of Tabuk, P.O. Box 4279, Tabuk 71491, Saudi Arabia \\ Correspondence should be addressed to Md. Nasiruzzaman; nasir3489@gmail.com \\ Received 9 September 2020; Revised 7 October 2020; Accepted 11 November 2020; Published 23 November 2020 \\ Academic Editor: Syed Abdul Mohiuddine
}

Copyright (c) $2020 \mathrm{Md}$. Nasiruzzaman and A. F. Aljohani. This is an open access article distributed under the Creative Commons Attribution License, which permits unrestricted use, distribution, and reproduction in any medium, provided the original work is properly cited.

The purpose of this article is to introduce a Kantorovich variant of Szász-Mirakjan operators by including the Dunkl analogue involving the Appell polynomials, namely, the Szász-Mirakjan-Jakimovski-Leviatan-type positive linear operators. We study the global approximation in terms of uniform modulus of smoothness and calculate the local direct theorems of the rate of convergence with the help of Lipschitz-type maximal functions in weighted space. Furthermore, the Voronovskaja-type approximation theorems of this new operator are also presented.

\section{Introduction}

In the year 1950, a famous mathematician Szász [1] invented the positive linear operators for the continuous function $f$ on $[0, \infty)$ and that were extensively searched rather than Bernstein operators [2]. For $z \in[0, \infty)$ and $f \in C[0, \infty)$, Szász introduced the operators as follows:

$$
S_{r}(f ; z)=e^{-r z} \sum_{k=0}^{\infty} \frac{(r z)^{k}}{k !} f\left(\frac{k}{r}\right)
$$

where $C[0, \infty)$ is the space of continuous functions on $[0, \infty)$. In recent years, Szász-Mirakjan operators were introduced by Sucu [3] by proposing an exponential function on Dunkl generalization by including a nonnegative number $\eta \geq 0$, such that

$$
\mathcal{S}_{r}^{*}(f ; z)=\frac{1}{e_{\eta}(r z)} \sum_{\kappa=0}^{\infty} \frac{(r z)^{\kappa}}{\gamma_{\eta}(\kappa)} f\left(\frac{\kappa+2 \eta \theta_{\kappa}}{r}\right)
$$

where $e_{\eta}(z)=\sum_{\kappa=0}^{\infty} z^{\kappa} / \gamma_{\eta}(\kappa)$ and a recursion formula for $s=0,1,2, \cdots$.

$$
\begin{aligned}
\frac{\gamma_{\eta}(\kappa+1)}{\left(\kappa+1+2 \eta \theta_{\kappa+1}\right)} & =\gamma_{\eta}(\kappa), \\
\theta_{\kappa} & = \begin{cases}0 & \text { if } \kappa=2 r, r \in \mathbb{N} \cup\{0\}, \\
1 & \text { if } \kappa=2 r+1, r \in \mathbb{N} \cup\{0\} .\end{cases}
\end{aligned}
$$

In 1969, Jakimovski and Leviatan introduced the sequence of Szász-Mirakjan-type positive linear operators by the use of Appell polynomials [4], $L(u) e^{u z}=\sum_{\kappa=0}^{\infty} H_{\kappa}(z) u^{\kappa}$ such that

$$
J_{r}(h ; z)=\frac{e^{-r z}}{L(1)} \sum_{\kappa=0}^{\infty} H_{\kappa}(r z) f\left(\frac{\kappa}{r}\right)
$$

where $L(1) \neq 0, L(u)=\sum_{\kappa=0}^{\infty} b_{\kappa} u^{\kappa}, H_{\kappa}(z)=\sum_{j=0}^{\kappa} b_{j}\left(z^{\kappa-j} /(\kappa-j)\right.$ !) $(\kappa \in \mathbb{N})$. Note that, if $L(1)=1$ in (4), the Szász-Mirakjan 
operator (1) is obtained. Most recently, in [5], Nasiruzzaman and Aljohani have introduced the Szász-Mirakjan-JakimovskiLeviatan-type operators involving the Dunkl generalization for the function $f \in C[0, \infty)$ by

$$
\mathscr{P}_{r, \eta}^{*}(f ; z)=\frac{1}{L(1) e_{\eta}(r z)} \sum_{\kappa=0}^{\infty} H_{\kappa}(r z) f\left(\frac{\kappa+2 \eta \theta_{\kappa}}{r}\right) .
$$

Lemma 1. [5]. For the test function $\gamma_{j}=t^{j}$, if $j=0,1,2,3,4$, the operators $\mathscr{P}_{r, \eta}^{*}$ have $\mathscr{P}_{r, \eta}^{*}\left(\gamma_{0} ; z\right)=1, \mathscr{P}_{r, \eta}^{*}\left(\gamma_{1} ; z\right)=z+(1 /$ $r)\left(\left(L^{\prime}(1) / L(1)\right)+2 \eta\right)$, and the following identities:

$$
\begin{aligned}
\mathscr{P}_{r, \eta}^{*}\left(\gamma_{2} ; z\right)= & z^{2}+\frac{1}{r}\left(\frac{2 L^{\prime}(1)}{L(1)}+4 \eta+1\right) z+\frac{1}{r^{2}} \\
& \cdot\left(\frac{L^{\prime \prime}(1)}{L(1)}+(1+4 \eta) \frac{L^{\prime}(1)}{L(1)}+4 \eta^{2}\right) \\
\mathscr{P}_{r, \eta}^{*}\left(\gamma_{3} ; z\right)= & z^{3}+\frac{3}{r}\left(\frac{L^{\prime}(1)}{L(1)}+2 \eta+1\right) z^{2}+\frac{1}{r^{2}} \\
& \cdot\left(\frac{3 L^{\prime \prime}(1)}{L(1)}+6(1+2 \eta) \frac{L^{\prime}(1)}{L(1)}+2+6 \eta+12 \eta^{2}\right) z+\frac{1}{r^{3}} \\
& \cdot\left(\frac{3 L^{\prime \prime \prime}(1)}{L(1)}+3(1+2 \eta) \frac{L^{\prime \prime}(1)}{L(1)}\right. \\
+ & \left.2\left(1+3 \eta+6 \eta^{2}\right) \frac{L^{\prime}(1)}{L(1)}+8 \eta^{3}\right) \\
& \left.\cdot \frac{L^{\prime \prime}(1)}{L(1)}+\left(6+16 \eta+24 \eta^{2}+32 \eta^{3}\right) \frac{L^{\prime}(1)}{L(1)}+16 \eta^{4}\right) \\
& \left.\cdot \frac{L^{\prime}(1)}{L(1)}+6+16 \eta+24 \eta^{+} 32 \eta^{3}\right) z+\frac{1}{r^{4}} \\
& \cdot\left(\frac{6 L^{\prime \prime}(1)}{L(1)}+8(1+3 \eta) \frac{L^{\prime}(1)}{L(1)}+11+24 \eta+24 \eta^{2}\right) z^{2} \\
\mathscr{P}_{r, \eta}^{*}\left(\gamma_{4} ; z\right)= & z^{4}+\frac{1}{r}\left(\frac{4 L^{\prime}(1)}{L(1)}+8 \eta+6\right) z^{3}+\frac{1}{r^{2}} \\
& (1) \\
&
\end{aligned}
$$

There are several research articles mentioned regarding the Szász-Mirakjan-type operators, for instance, [6-13]. For some further related concepts and approximation, we refer to see $[9,10,14-20]$.

\section{Kantorovich Operators Involving Appell Polynomials and Their Moments}

In this section, we construct the generalized operators of recent investigation [5] including the Kantorovich polyno- mial. For this purpose, we let $f \in C_{\Phi}[0, \infty)=\{f \in C[0$, $\left.\infty): f(t)=O\left(t^{\Phi}\right)\right\}$ as $t \rightarrow \infty$; then, for all $z \in[0, \infty), \Phi>$ $r, r \in \mathbb{N}, L(1) \neq 0$, and $\eta \geq 0$, we define the operators as follows:

$$
\mathscr{R}_{r, \eta}^{*}(f ; z)=\frac{r}{L(1) e_{\eta}(r z)} \sum_{\kappa=0}^{\infty} H_{\kappa}(r z) \int_{\left(\kappa+2 \eta \theta_{\kappa}\right) / r}^{\left(\kappa+1+2 \eta \theta_{\kappa}\right) / r} f(t) \mathrm{d} t .
$$

Lemma 2. For $j=0,1,2,3$, 4, let the test function be $\gamma_{j}=t^{j}$. Then, operators $\mathscr{R}_{r, \eta}^{*}(\cdot ; \cdot)$ have the following identities:

$$
\mathscr{R}_{r, \eta}^{*}\left(\gamma_{0} ; z\right)=1
$$

$$
\begin{aligned}
& \mathscr{R}_{r, \eta}^{*}\left(\gamma_{1} ; z\right)=z+\frac{1}{2 r}\left(2 \frac{L^{\prime}(1)}{L(1)}+4 \eta+1\right), \\
& \mathscr{R}_{r, \eta}^{*}\left(\gamma_{2} ; z\right)=z^{2}+\frac{1}{r}\left(2 \frac{L^{\prime}(1)}{L(1)}+4 \eta+2\right) z+\frac{1}{3 r^{2}} \\
& \cdot\left(3 \frac{L^{\prime \prime}(1)}{L(1)}+6(1+2 \eta) \frac{L^{\prime}(1)}{L(1)}+12 \eta^{2}+6 \eta+1\right) \text {, } \\
& \mathscr{R}_{r, \eta}^{*}\left(\gamma_{3} ; z\right)=z^{3}+\frac{3}{2 r}\left(2 \frac{L^{\prime}(1)}{L(1)}+4 \eta+3\right) z^{2}+\frac{3}{2 r^{2}} \\
& \cdot\left(2 \frac{L^{\prime \prime}(1)}{L(1)}+2(3+4 \eta) \frac{L^{\prime}(1)}{L(1)}+8 \eta^{2}+8 \eta+3\right) z \\
& +\frac{1}{4 r^{3}}\left(12 \frac{L^{\prime \prime \prime}(1)}{L(1)}+6(3+4 \eta) \frac{L^{\prime \prime}(1)}{L(1)}+6\right. \\
& \left.\cdot\left(8 \eta^{2}+8 \eta+3\right) \frac{L^{\prime}(1)}{L(1)}+32 \eta^{3}+8 \eta^{2}+8 \eta+1\right) \text {, } \\
& \mathscr{R}_{r, \eta}^{*}\left(\gamma_{4} ; z\right)=z^{4}+\frac{1}{r}\left(4 \frac{L^{\prime}(1)}{L(1)}+8 \eta+8\right) z^{3}+\frac{1}{r^{2}} \\
& \cdot\left(6 \frac{L^{\prime \prime}(1)}{L(1)}+(14+24 \eta) \frac{L^{\prime}(1)}{L(1)}+24 \eta^{2}+36 \eta+9\right) z^{2} \\
& +\frac{1}{r^{3}}\left(4 \frac{L^{\prime \prime \prime}(1)}{L(1)}+(14+24 \eta) \frac{L^{\prime \prime}(1)}{L(1)}\right. \\
& \left.+\left(38+72 \eta+48 \eta^{2}\right) \frac{L^{\prime}(1)}{L(1)}+32 \eta^{3}+48 \eta^{2}+36 \eta+13\right) z \\
& +\frac{1}{r^{4}}\left(\frac{L^{\prime} v(1)}{L(1)}+4(3+2 \eta) \frac{L^{\prime \prime \prime}(1)}{L(1)}+\left(19+36 \eta+24 \eta^{2}\right)\right. \\
& \cdot \frac{L^{\prime \prime}(1)}{L(1)}+\left(13+36 \eta+48 \eta^{2}+32 \eta^{3}\right) \frac{L^{\prime}(1)}{L(1)}+16 \eta^{4} \\
& \left.+16 \eta^{3}+8 \eta^{2}+2 \eta+1\right) \text {. }
\end{aligned}
$$

Proof. To prove this Lemma, we take into account [5] Lemma 1. Thus, for all $j=0,1,2,3,4$ and $\gamma_{j}=t^{j}$, we can conclude that 


$$
\int_{\left(\kappa+2 \eta \theta_{\kappa}\right) / r}^{\left(\kappa+1+2 \eta \theta_{\kappa}\right) / r} t^{j} \mathrm{~d} t=\left\{\begin{array}{lr}
\frac{1}{r} & \text { for } j=0, \\
\frac{1}{2 r^{2}}+\frac{1}{r}\left(\frac{\kappa+2 \eta \theta_{\kappa}}{r}\right) & \text { for } j=1, \\
\frac{1}{3 r^{3}}+\frac{1}{r^{2}}\left(\frac{\kappa+2 \eta \theta_{\kappa}}{r}\right)+\frac{1}{r}\left(\frac{\kappa+2 \eta \theta_{\kappa}}{r}\right)^{2} & \text { for } j=2, \\
\frac{1}{4 r^{4}}+\frac{1}{r^{3}}\left(\frac{\kappa+2 \eta \theta_{\kappa}}{r}\right)+\frac{3}{2 r^{2}}\left(\frac{\kappa+2 \eta \theta_{\kappa}}{r}\right)^{2}+\frac{1}{r}\left(\frac{\kappa+2 \eta \theta_{\kappa}}{r}\right)^{3} & \text { for } j=3, \\
\frac{1}{5 r^{5}}+\frac{1}{r^{4}}\left(\frac{\kappa+2 \eta \theta_{\kappa}}{r}\right)+\frac{2}{r^{3}}\left(\frac{\kappa+2 \eta \theta_{\kappa}}{r}\right)^{2}+\frac{2}{r^{2}}\left(\frac{\kappa+2 \eta \theta_{\kappa}}{r}\right)^{3}+\frac{1}{r}\left(\frac{\kappa+2 \eta \theta_{\kappa}}{r}\right)^{4} & \text { for } j=4 .
\end{array}\right.
$$

Thus, from (7) and (9), clearly we can write

$$
\begin{aligned}
\mathscr{R}_{r, \eta}^{*}\left(\gamma_{0} ; z\right)= & \mathscr{P}_{r, \eta}^{*}\left(\gamma_{0} ; z\right)=1, \\
\mathscr{R}_{r, \eta}^{*}\left(\gamma_{1} ; z\right)= & \mathscr{P}_{r, \eta}^{*}\left(\gamma_{1} ; z\right)+\frac{1}{2 r} \mathscr{P}_{r, \eta}^{*}\left(\gamma_{0} ; z\right), \\
\mathscr{R}_{r, \eta}^{*}\left(\gamma_{2} ; z\right)= & \mathscr{P}_{r, \eta}^{*}\left(\gamma_{2} ; z\right)+\frac{1}{r} \mathscr{P}_{r, \eta}^{*}\left(\gamma_{1} ; z\right)+\frac{1}{3 r^{2}} \mathscr{P}_{r, \eta}^{*}\left(\gamma_{0} ; z\right), \\
& +\frac{1}{4 r^{3}} \mathscr{P}_{r, \eta}^{*}\left(\gamma_{0} ; z\right), \\
\mathscr{R}_{r, \eta}^{*}\left(\gamma_{3} ; z\right)= & \mathscr{P}_{r, \eta}^{*}\left(\gamma_{3} ; z\right)+\frac{3}{2 r} \mathscr{P}_{r, \eta}^{*}\left(\gamma_{2} ; z\right)+\frac{1}{r^{2}} \mathscr{P}_{r, \eta}^{*}\left(\gamma_{1} ; z\right) \\
\mathscr{R}_{r, \eta}^{*}\left(\gamma_{4} ; z\right)= & \mathscr{P}_{r, \eta}^{*}\left(\gamma_{4} ; z\right)+\frac{2}{r} \mathscr{P}_{r, \eta}^{*}\left(\gamma_{3} ; z\right)+\frac{2}{r^{2}} \mathscr{P}_{r, \eta}^{*}\left(\gamma_{2} ; z\right) \\
& +\frac{1}{r^{3}} \mathscr{P}_{r, \eta}^{*}\left(\gamma_{1} ; z\right)+\frac{1}{r^{4}} \mathscr{P}_{r, \eta}^{*}\left(\gamma_{0} ; z\right) .
\end{aligned}
$$
results.

Therefore, by applying Lemma 1, we get the required

Lemma 3. For the central moments $\left(\gamma_{1}-z\right)^{i}, i=1,2,4$, we have the following identities:

$$
\begin{aligned}
\mathscr{R}_{r, \eta}^{*}\left(\left(\gamma_{1}-z\right) ; z\right)= & \frac{1}{2 r}\left(2 \frac{L^{\prime}(1)}{L(1)}+4 \eta+1\right) \\
\mathscr{R}_{r, \eta}^{*}\left(\left(\gamma_{1}-z\right)^{2} ; z\right)= & \frac{z}{r}+\frac{1}{3 r^{2}}\left(3 \frac{L^{\prime \prime}(1)}{L(1)}+6(1+2 \eta) \frac{L^{\prime}(1)}{L(1)}\right. \\
& \left.+12 \eta^{2}+6 \eta+1\right)
\end{aligned}
$$

$$
\begin{aligned}
\mathscr{R}_{r, \eta}^{*}\left(\left(\gamma_{1}-z\right)^{4} ; z\right)= & -\frac{7}{r^{2}} z^{2}+\frac{1}{r^{3}}\left(-8 \frac{L^{\prime \prime \prime}(1)}{L(1)}-4 \frac{L^{\prime \prime}(1)}{L(1)}\right. \\
& \left.+(24 \eta+20) \frac{L^{\prime}(1)}{L(1)}+40 \eta^{2}+28 \eta+12\right) z \\
& +\frac{1}{r^{4}}\left(\frac{L^{\prime} v(1)}{L(1)}+4(3+2 \eta) \frac{L^{\prime \prime \prime}(1)}{L(1)}\right. \\
& +\left(19+36 \eta+24 \eta^{2}\right) \frac{L^{\prime \prime}(1)}{L(1)}+(13+36 \eta \\
& \left.+48 \eta^{2}+32 \eta^{3}\right) \frac{L^{\prime}(1)}{L(1)}+16 \eta^{4}+16 \eta^{3} \\
& \left.+8 \eta^{2}+2 \eta+1\right) .
\end{aligned}
$$

\section{Approximations in Weighted Space}

In the present section, we follow the well-known results by Gadziev [21] and recall the results in weighted spaces with some additional conditions precisely, under the analogous of P.P. Korovkin's theorem holds. In order to define the uniformly approximations, we take $z \rightarrow \varphi(z)$ be the kind of functions which is continuous and strictly increasing with the assumptions $\Phi(z)=1+\varphi^{2}(z)$ and $\lim _{z \rightarrow \infty} \Phi(z)=\infty$. For this reason, we let $B_{\Phi}[0, \infty)$ be a set of all such functions which are defined on $[0, \infty)$ and verifying the results

$$
B_{\Phi}[0, \infty)=\left\{f:|f(z)| \leq K_{f} \Phi(z)\right\}
$$

where $K_{f}$ is a constant and depending only on function $f$ and $B_{\Phi}[0, \infty)$ equipped the norm with

$$
\|f\|_{\Phi}=\sup _{z \in[0, \infty)} \frac{|f(z)|}{\Phi(z)} .
$$


Furthermore, we denote the set all continuous functions on $[0, \infty)$ by $C[0, \infty)$ and its subsets be $C_{\Phi}[0, \infty)$ defined as $C_{\Phi}[0, \infty)=B_{\Phi}[0, \infty) \cap C[0, \infty)$.

It is well known for the sequence of linear positive operators $\left\{K_{r}\right\}_{r \geq 1}$ (see [21]) maps $C_{\Phi}[0, \infty)$ into $B_{\Phi}[0, \infty)$ if and only if

$$
\left|K_{r}(\Phi ; z)\right| \leq M \Phi(z)
$$

where $M$ is a positive constant. For $m \in \mathbb{N}$, let us denote

$C_{\Phi}^{m}[0, \infty)=\left\{f \in C_{\Phi}[0, \infty): \lim _{z \rightarrow \infty} \frac{f(z)}{\Phi(z)}=c\right.$, exists and is finite $\}$.

Theorem 4. Let $H_{f}=\{f:$ such that $f(z) / \Phi(z)$ is convergent when $z \rightarrow \infty\}$. Then, for every $f \in H_{f} \cap C_{\Phi}[0, \infty)$, operators (7) are uniformly convergent on each compact subset of $[0$, $\infty)$ such that

$$
\mathscr{R}_{r, \eta}^{*}(f ; z) \Rightarrow f,
$$

where $\Rightarrow$ denotes the uniform convergence.

Proof. In view of Lemma 2, we use Korovkin's theorem by [22]; then, it is enough to see that for each $j=0,1,2$

$$
\mathscr{R}_{r, \eta}^{*}\left(\gamma_{j} ; z\right) \rightarrow z^{j}
$$

uniformly. Thus obviously, we get $\lim _{r \rightarrow \infty} \mathscr{R}_{r, \eta}^{*}\left(\gamma_{0} ; z\right)=1$, $\lim _{r \rightarrow \infty} \mathscr{R}_{r, \eta}^{*}\left(\gamma_{1} ; z\right)=z$, and $\lim _{r \rightarrow \infty} \mathscr{R}_{r, \eta}^{*}\left(\gamma_{2} ; z\right)=z^{2}$, which completes the proof of Theorem 4 .

Theorem $5[21,23]$. Let the positive linear operators $\left\{J_{r}\right\}_{r \geq 1}$ acting from $C_{\Phi}[0, \infty)$ to $B_{\Phi}[0, \infty)$ and for $j=0,1,2$ if it verifies that $\lim _{r \rightarrow \infty}\left\|J_{r}\left(\gamma_{j}\right)-z^{j}\right\|_{\Phi}=0$, then for every $f \in C_{\Phi}^{m}[0$, $\infty)$ it satisfies

$$
\lim _{r \rightarrow \infty}\left\|J_{r}(f)-f\right\|_{\Phi}=0
$$

Theorem 6. For every $\varphi \in C_{\Phi}^{m}[0, \infty)$, operators $\mathscr{R}_{r, \eta}^{*}$ satisfy

$$
\lim _{r \rightarrow \infty}\left\|\mathscr{R}_{r, \eta}^{*}(\varphi)-\varphi\right\|_{\Phi}=0
$$

Proof. It is enough to prove Theorem 6; we use the wellknown Korovkin theorem and show

$$
\lim _{r \rightarrow \infty}\left\|\mathscr{R}_{r, \eta}^{*}\left(\gamma_{j}\right)-z^{j}\right\|_{\Phi}=0, \quad j=0,1,2 .
$$

Taking into account Lemma 2, then it is easy to see that

$$
\left\|\mathscr{R}_{r, \eta}^{*}\left(\gamma_{0}\right)-1\right\|_{\Phi}=\sup _{z \in[0, \infty)} \frac{\left|\mathscr{R}_{r, \eta}^{*}(1 ; z)-1\right|}{\Phi(z)}=0
$$

For $j=1$, we can write here

$$
\begin{aligned}
\left\|\mathscr{R}_{r, \eta}^{*}\left(\gamma_{1}\right)-z\right\|_{\Phi} & =\sup _{z \in[0, \infty)} \frac{\left|\mathscr{R}_{r, \eta}^{*}\left(\gamma_{1} ; z\right)-z\right|}{\Phi(z)} \\
& =\sup _{z \in[0, \infty)} \frac{1}{\Phi(z)}\left|\frac{1}{2 r}\left(\frac{2 L^{\prime}(1)}{\mathrm{L}(1)}+4 \eta+1\right)\right| .
\end{aligned}
$$

If $r \rightarrow \infty$, then easily we get $\left\|\mathscr{R}_{r, \eta}^{*}\left(\gamma_{1}\right)-z\right\|_{\Phi} \rightarrow 0$. Similarly, for $j=2$, we conclude that

$$
\begin{aligned}
\left\|\mathscr{R}_{r, \eta}^{*}\left(\gamma_{2}\right)-z^{2}\right\|_{\Phi}= & \sup _{z \in[0, \infty)} \frac{\left|\mathscr{R}_{r, \eta}^{*}\left(\gamma_{2} ; z\right)-z^{2}\right|}{\Phi(z)} \\
= & \sup _{z \in[0, \infty)} \frac{z}{1+z^{2}}\left|\frac{1}{r}\left(2 \frac{L^{\prime}(1)}{L(1)}+4 \eta+2\right)\right| \\
& +\sup _{z \in[0, \infty)} \frac{1}{\Phi(z)} \mid \frac{1}{3 r^{2}}\left(3 \frac{L^{\prime \prime}(1)}{L(1)}+6(1+2 \eta)\right. \\
& \left.\cdot \frac{L^{\prime}(1)}{L(1)}+12 \eta^{2}+6 \eta+1\right) .
\end{aligned}
$$

Thus, we easily get $\left\|\mathscr{R}_{r, \eta}^{*}\left(\gamma_{2}\right)-z^{2}\right\|_{\Phi} \rightarrow 0$, as $r \rightarrow \infty$.

Theorem 7. If $\varphi \in C_{\Phi}^{m}[0, \infty)$. Then, operators $\mathscr{R}_{r, \eta}^{*}$ follow that

$$
\lim _{r \rightarrow \infty} \sup _{z \in[0, \infty)} \frac{\left|\mathscr{R}_{r, \eta}^{*}(\varphi ; z)-\varphi(z)\right|}{(\Phi(z))^{1+\xi}}=0,
$$

where the number $\xi \in[0, \infty)$.

Proof. By the virtue of $|\varphi(z)| \leq\|\varphi\|_{\Phi}\left(1+z^{2}\right)$ and for any positive real $z_{0}$, we easily obtain

$$
\begin{aligned}
& \lim _{r \rightarrow \infty} \sup _{z \in[0, \infty)} \frac{\left|\mathscr{R}_{r, \eta}^{*}(\varphi ; z)-\varphi(z)\right|}{(\Phi(z))^{1+\xi}} \\
& \leq \sup _{z \leq z_{0}} \frac{\left|\mathscr{R}_{r, \eta}^{*}(\varphi ; z)-\varphi(z)\right|}{(\Phi(z))^{1+\xi}}+\sup _{z \geq z_{0}} \frac{\left|\mathscr{R}_{r, \eta}^{*}(\varphi ; z)-\varphi(z)\right|}{(\Phi(z))^{1+\xi}} \\
& \leq\left\|\mathscr{R}_{r, \eta}^{*}(\varphi ; z)-\varphi(z)\right\|_{C\left[0, z_{0}\right]}+\|\varphi\|_{\Phi} \sup _{z \geq z_{0}} \\
& \times \frac{\left|\mathscr{R}_{r, \eta}^{*}\left(1+t^{2} ; z\right)-\varphi(z)\right|}{(\Phi(z))^{1+\xi}}+\sup _{z \geq z_{0}} \frac{|\varphi(z)|}{(\Phi(z))^{1+\xi}} \\
& =\mathscr{F}_{1}+\mathscr{J}_{2}+\mathscr{f}_{3} \text {, (suppose). }
\end{aligned}
$$

Thus,

$$
\mathscr{F}_{3}=\sup _{z \geq z_{0}} \frac{|\varphi(z)|}{(\Phi(z))^{1+\xi}} \leq \sup _{z \geq z_{0}} \frac{\|\varphi\|_{\Phi}\left(1+z^{2}\right)}{(\Phi(z))^{1+\xi}} \leq \frac{\|\varphi\|_{\Phi}}{\left(1+z_{0}^{2}\right)^{\xi}} .
$$


From Lemma 2, it follows that

$$
\lim _{r \rightarrow \infty} \sup _{z \geq z_{0}} \frac{\mathscr{R}_{r, \eta}^{*}\left(1+t^{2} ; z\right)}{\Phi(z)}=1 .
$$
that

Now, for each $\varepsilon>0$, there exists $r_{1} \in \mathbb{N}$ for all $r \geq r_{1}$ such

$$
\sup _{z \geq z_{0}} \frac{\mathscr{R}_{r, \eta}^{*}\left(1+t^{2} ; z\right)}{\Phi(z)} \leq \frac{\left(1+z_{0}^{2}\right)^{\xi}}{\|\varphi\|_{\Phi}} \frac{\varepsilon}{3}+1 .
$$

Therefore, for all $r \geq r_{1}$

$$
\mathscr{J}_{2}=\|\varphi\|_{\Phi} \sup _{z \geq z_{0}} \frac{\mathscr{R}_{r, \eta}^{*}\left(1+t^{2} ; z\right)}{(\Phi(z))^{1+\xi}} \leq \frac{\|\varphi\|_{\Phi}}{\left(1+z_{0}^{2}\right)^{\xi}}+\frac{\varepsilon}{3}
$$

In view of (26) and (29), we get

$$
\mathscr{J}_{2}+\mathscr{J}_{3} \leq 2 \frac{\|\varphi\|_{\Phi}}{\left(1+z_{0}^{2}\right)^{\xi}}+\frac{\varepsilon}{3} \text {. }
$$

If we choose any $z_{0}$ so large, such that $\|\varphi\|_{\Phi} /\left(1+z_{0}^{2}\right)^{\xi} \leq$ $\varepsilon / 6$, then we get

$$
\mathscr{J}_{2}+\mathscr{J}_{3} \leq \frac{2 \varepsilon}{3}, \quad \text { for all } r \geq r_{1}
$$

On the other hand, there exists $r_{2} \geq r$ such that

$$
\mathscr{J}_{1}=\left\|\mathscr{R}_{r, \eta}^{*}(\varphi ; z)-\varphi(z)\right\|_{C\left[0, z_{0}\right]} \leq \frac{\varepsilon}{3}
$$

Finally, take $r_{3}=\max \left(r_{1}, r_{2}\right)$ and on combining (31) and (32) with the above expression, we get

$$
\sup _{z \in[0, \infty)} \frac{\left|\mathscr{R}_{r, \eta}^{*}(\varphi ; z)-\varphi(z)\right|}{(\Phi(z))^{1+\xi}}<\varepsilon .
$$

This completes the proof of Theorem 7 .

Definition 8. For every $\bar{\delta}>0$ and all $f \in C[0, \infty)$, the modulus of continuity of the uniformly continuous function $f$ on $[0$, $\infty)$ defined as

$$
\begin{aligned}
\bar{\omega}(f ; \bar{\delta}) & =\sup _{\left|t_{1}-t_{2}\right| \leq \bar{\delta}}\left|f\left(t_{1}\right)-f\left(t_{2}\right)\right|, t_{1}, t_{2} \in[0, \infty), \\
\left|f\left(t_{1}\right)-f\left(t_{2}\right)\right| & \leq\left(1+\frac{\left|t_{1}-t_{2}\right|}{\bar{\delta}^{2}}\right) \bar{\omega}(f ; \bar{\delta}) .
\end{aligned}
$$

Theorem 9 [24]. Let the sequence of positive linear operators $\{K\}_{r \geq 1}:[x, y] \rightarrow C[u, v]$ and $[u, v] \subseteq[x, y]$, then
(1) for any $f \in C[x, y]$ and $z \in[u, v]$, it follows that

$$
\begin{aligned}
& \left|K_{r}(f ; z)-f(z)\right| \\
& \quad \leq|f(z)|\left|K_{r}(1 ; z)-1\right| \\
& \quad+\left\{K_{r}(1 ; z)+\frac{1}{\bar{\delta}} \sqrt{K_{r}\left((t-z)^{2} ; z\right)} \sqrt{K_{r}(1 ; z)}\right\} \bar{\omega}(f ; \bar{\delta}),
\end{aligned}
$$

(2) if any $\varphi^{\prime} \in C[x, y]$, then for all $z \in[u, v]$ one has

$$
\begin{aligned}
& \left|K_{r}(\phi ; z)-\phi(z)\right| \\
& \leq \mid \\
& \quad \times|(z)|\left|K_{r}(1 ; z)-1\right|+\left|\phi_{r}(t-z ; z)\right|+K_{r}\left((t-z)^{2} ; z\right) \\
& \quad \times\left\{\sqrt{K_{r}(1 ; z)}+\frac{1}{\bar{\delta}} \sqrt{K_{r}\left((t-z)^{2} ; z\right)}\right\} \bar{\omega}\left(\phi^{\prime} ; \bar{\delta}\right) .
\end{aligned}
$$

Theorem 10. Let $f \in C_{\Phi}[0, \infty)$, then for all $z \in[0, \infty)$ it follows the inequality

$$
\left|\mathscr{R}_{r, \eta}^{*}(f ; z)-f(z)\right| \leq 2 \bar{\omega}\left(f ; \sqrt{\bar{\delta}_{r, \eta}^{*}(z)}\right),
$$

where $\bar{\delta}=\sqrt{\bar{\delta}_{r, \eta}^{*}(z)}=\sqrt{\mathscr{R}_{r, \eta}^{*}\left(\left(\gamma_{1}-z\right)^{2} ; z\right)}$.

Proof. If we consider Lemma 2 and Theorem 9, then we can obtain

$$
\begin{aligned}
& \left|\mathscr{R}_{r, \eta}^{*}(f ; z)-f(z)\right| \\
& \leq|f(z)|\left|\mathscr{R}_{r, \eta}^{*}\left(\gamma_{0} ; z\right)-1\right| \\
& \quad+\left\{\mathscr{R}_{r, \eta}^{*}\left(\gamma_{0} ; z\right)+\frac{1}{\bar{\delta}} \sqrt{\mathscr{R}_{r, \eta}^{*}\left(\left(\gamma_{0}-z\right)^{2} ; z\right)} \sqrt{\mathscr{R}_{r, \eta}^{*}\left(\gamma_{0} ; z\right)}\right\} \bar{\omega} \\
& \quad \times(f ; \bar{\delta}),
\end{aligned}
$$

where if we take $\bar{\delta}=\sqrt{\bar{\delta}_{r, \eta}^{*}(z)}=\sqrt{\mathscr{R}_{r, \eta}^{*}\left(\left(\gamma_{1}-z\right)^{2} ; z\right)}$ then we are easily denumerable to get results.

Theorem 11. For any $z \in[0, \infty)$, if $\phi \in C_{\Phi}^{\prime}[0, \infty)$, then we have the inequality

$$
\begin{aligned}
\left|\mathscr{R}_{r, \eta}^{*}(\phi ; z)-\phi(z)\right| \leq & \frac{1}{2 r}\left(2 \frac{L^{\prime}(1)}{L(1)}+4 \eta+1\right)|| \phi^{\prime}(z) \mid \\
& +2 \bar{\delta}_{r, \eta}^{*}(z) \bar{\omega}\left(\phi^{\prime} ; \sqrt{\bar{\delta}_{r, \eta}^{*}(z)}\right),
\end{aligned}
$$

where $\bar{\delta}=\sqrt{\bar{\delta}_{r, \eta}^{*}(z)}=\sqrt{\mathscr{R}_{r, \eta}^{*}\left(\left(\gamma_{1}-z\right)^{2} ; z\right)}$. 
Proof. If we consider Lemmas 2 and 3 and (2) of Theorem 9, then it is obvious to get that

$$
\begin{aligned}
& \left|\mathscr{R}_{r, \eta}^{*}(\phi ; z)-\phi(z)\right| \\
& \leq\left|\mathscr{R}_{r, \eta}^{*}\left(\gamma_{0} ; z\right)-1\right||\phi(z)|+\left|\phi^{\prime}(z)\right| \\
& \quad \times\left|\mathscr{R}_{r, \eta}^{*}\left(\gamma_{1}-z ; z\right)\right|+\mathscr{R}_{r, \eta}^{*}\left(\left(\gamma_{1}-z\right)^{2} ; z\right) \\
& \quad \times\left\{\sqrt{\mathscr{R}_{r, \eta}^{*}\left(\gamma_{0} ; z\right)}+\frac{1}{\bar{\delta}} \sqrt{\mathscr{R}_{r, \eta}^{*}\left(\left(\gamma_{1}-z\right)^{2} ; z\right)}\right\} \bar{\omega}\left(\phi^{\prime} ; \bar{\delta}\right) .
\end{aligned}
$$

Put $\bar{\delta}=\sqrt{\overline{\delta_{r, \eta}^{*}(z)}}=\sqrt{\mathscr{R}_{r, \eta}^{*}\left(\left(\gamma_{1}-z\right)^{2} ; z\right)}$, then we easily get our desired results of Theorem 11 .

From [25] for an arbitrary $f \in C_{\Phi}^{m}[0, \infty), m \in \mathbb{N} \cup\{0\}$, the weighted modulus of continuity introduced such that

$$
\bar{\Omega}(f ; \bar{\delta})=\sup _{z \in[0, \infty),|h| \leq \bar{\delta}} \frac{|f(z+h)-f(z)|}{\left(1+h^{2}\right)\left(1+z^{2}\right)} .
$$

Two main properties of this modulus of continuity are $\lim _{\bar{\delta} \rightarrow 0} \bar{\Omega}(f ; \bar{\delta})=0$ and

$$
\begin{aligned}
&|f(t)-f(z)| \leq 2\left(1+\frac{|t-z|}{\bar{\delta}}\right)\left(1+\bar{\delta}^{2}\right)\left(1+z^{2}\right) \\
& \cdot\left(1+(t-z)^{2}\right) \bar{\Omega}(f ; \bar{\delta})
\end{aligned}
$$

where $t, z \in[0, \infty)$ and $\bar{\Omega}$ weighted modulus of continuity of the function for $f \in C_{\Phi}^{m}[0, \infty)$.

Theorem 12. Let $f \in C_{\Phi}^{m}[0, \infty)$, then for all $z \in[0, \infty)$ we have the inequality

$$
\sup _{z \in\left[0, \alpha_{r}(\eta)\right)} \frac{\left|\mathscr{R}_{r, \eta}^{*}(f ; z)-f(z)\right|}{1+z^{2}} \leq M\left(1+\alpha_{r}(\eta)\right) \Omega\left(f ; \sqrt{\alpha_{r}(\eta)}\right),
$$

where $M=2\left(2+M_{1}+\sqrt{M_{2}}\right)>0$, for $M_{1}, M_{2}>0$ and

$$
\begin{aligned}
\alpha_{r}(\eta)= & \max \left\{\frac{1}{r}, \frac{1}{3 r^{2}}\left(3 \frac{L^{\prime \prime}(1)}{L(1)}+6(1+2 \eta) \frac{L^{\prime}(1)}{L(1)}\right.\right. \\
& \left.\left.+12 \eta^{2}+6 \eta+1\right)\right\} .
\end{aligned}
$$

Proof. We use expressions (41) and (42) and applying the Cauchy-Schwarz inequality to operators $\mathscr{R}_{r, \eta}^{*}$, we get

$$
\begin{aligned}
\left|\mathscr{R}_{r, \eta}^{*}(f ; z)-f(z)\right| \leq & 2\left(1+\bar{\delta}^{2}\right)\left(1+z^{2}\right) \bar{\Omega}(f ; \bar{\delta}) \\
& \cdot\left\{1+\mathscr{R}_{r, \eta}^{*}\left(\left(\gamma_{1}-z\right)^{2} ; z\right)+\mathscr{R}_{r, \eta}^{*}\right. \\
& \left.\cdot\left(\left(1+\left(\gamma_{1}-z\right)^{2}\right) \frac{\left|\gamma_{1}-z\right|}{\bar{\delta}} ; z\right)\right\} .
\end{aligned}
$$

We know the expression

$$
\begin{aligned}
\mathscr{R}_{r, \eta}^{*}( & \left.\left(1+\left(\gamma_{1}-z\right)^{2}\right) \frac{\left|\gamma_{1}-z\right|}{\bar{\delta}} ; z\right) \\
= & \frac{1}{\bar{\delta}} \mathscr{R}_{r, \eta}^{*}\left(\left|\gamma_{1}-z\right| ; z\right)+\mathscr{R}_{r, \eta}^{*}\left(\left(\gamma_{1}-z\right)^{2} \frac{\left|\gamma_{1}-z\right|}{\bar{\delta}} ; z\right) \\
\leq & \frac{1}{\bar{\delta}}\left(\mathscr{R}_{r, \eta}^{*}\left(\gamma_{1}-z\right)^{2} ; z\right)^{1 / 2}+\left(\mathscr{R}_{r, \eta}^{*}\left(\left(\gamma_{1}-z\right)^{4} ; z\right)\right)^{1 / 2} \\
& \times\left\{\mathscr{R}_{r, \eta}^{*}\left(\frac{\left(\gamma_{1}-z\right)^{2}}{\bar{\delta}^{2}} ; z\right)\right\}^{1 / 2} \\
= & \frac{1}{\bar{\delta}}\left(\mathscr{R}_{r, \eta}^{*}\left(\gamma_{1}-z\right)^{2} ; z\right)^{1 / 2}\left\{1+\sqrt{\mathscr{R}_{r, \eta}^{*}\left(\left(\gamma_{1}-z\right)^{4} ; z\right)}\right\} .
\end{aligned}
$$

In view of Lemma 3, we can obtain

$$
\begin{aligned}
\mathscr{R}_{r, \eta}^{*}\left(\left(\gamma_{1}-z\right)^{2} ; z\right) & \leq \alpha_{r}(\eta)(z+1) \leq M_{1}(z+1) \text { as } r \rightarrow \infty, \\
\mathscr{R}_{r, \eta}^{*}\left(\left(\gamma_{1}-z\right)^{4} ; z\right) & \leq \beta_{r}(\eta)\left(z^{2}+z+1\right) \\
& \leq M_{2}\left(z^{2}+z+1\right) \text { as } r \rightarrow \infty,
\end{aligned}
$$

where $M_{1}$ and $M_{2}$ are positive constant and

$$
\begin{aligned}
\alpha_{r}(\eta)= & \max \left\{\frac{1}{r}, \frac{1}{3 r^{2}}\left(3 \frac{L^{\prime \prime}(1)}{L(1)}+6(1+2 \eta) \frac{L^{\prime}(1)}{L(1)}\right.\right. \\
& \left.\left.+12 \eta^{2}+6 \eta+1\right)\right\}, \\
\beta_{r}(\eta)= & \max \left\{-\frac{7}{r^{2}}, \frac{1}{r^{3}}\left(-8 \frac{L^{\prime \prime \prime}(1)}{L(1)}-4 \frac{L^{\prime \prime}(1)}{L(1)}+(24 \eta+20)\right.\right. \\
& \left.\cdot \frac{L^{\prime}(1)}{L(1)}+40 \eta^{2}+28 \eta+12\right), \frac{1}{r^{4}}\left(\frac{L^{\prime} v(1)}{L(1)}+4(3+2 \eta)\right. \\
& \cdot \frac{L^{\prime \prime \prime}(1)}{L(1)}+\left(19+36 \eta+24 \eta^{2}\right) \frac{L^{\prime \prime}(1)}{L(1)} \\
& +\left(13+36 \eta+48 \eta^{2}+32 \eta^{3}\right) \frac{L^{\prime}(1)}{L(1)}+16 \eta^{4}+16 \eta^{3} \\
& \left.\left.+8 \eta^{2}+2 \eta+1\right)\right\} .
\end{aligned}
$$


Thus, from inequality (45), we get

$$
\begin{aligned}
& \left|\mathscr{R}_{r, \eta}^{*}(f ; z)-f(z)\right| \\
& \leq 2\left(1+\bar{\delta}^{2}\right)\left(1+z^{2}\right) \bar{\Omega}(f ; \bar{\delta})\left[1+\mathscr{R}_{r, \eta}^{*}\left(\left(\gamma_{1}-z\right)^{2} ; z\right)\right. \\
& \left.\quad+\frac{1}{\bar{\delta}}\left(\mathscr{R}_{r, \eta}^{*}\left(\gamma_{1}-z\right)^{2} ; z\right)^{1 / 2}\left\{1+\sqrt{\mathscr{R}_{r, \eta}^{*}\left(\left(\gamma_{1}-z\right)^{4} ; z\right)}\right\}\right] \\
& \leq 2\left(1+\bar{\delta}^{2}\right)\left(1+z^{2}\right) \bar{\Omega}(f ; \bar{\delta})\left[1+M_{1}(z+1)\right. \\
& \left.\quad+\frac{1}{\bar{\delta}} \sqrt{\alpha_{r}(\eta)(z+1)}\left\{1+\sqrt{M_{2}\left(z^{2}+z+1\right)}\right\}\right] .
\end{aligned}
$$

If we choose $\bar{\delta}=\sqrt{\alpha_{r}(\eta)}$ and taking supremum $z \in[0$, $\left.\alpha_{r}(\eta)\right)$, then we easily get the result.

\section{Direct Approximation Results of $\mathscr{R}_{r, \eta}^{*}$}

The present section gives some direct approximation results in space of $K$-functional and in Lipschitz spaces. We take $C_{b}[0, \infty)$ be the set of all continuous and bounded functions defined on $[0, \infty)$.

Definition 13. For every $\bar{\delta}>0$ and $f \in C[0, \infty)$ the $K$-functional is defined such that

$$
\begin{aligned}
& \mathscr{K}_{\psi}(f ; \bar{\delta}) \\
& \quad=\inf \left\{\left(\|f-\psi\|_{C_{b}[0, \infty)}+\bar{\delta}\left\|\psi^{\prime \prime}\right\|_{C_{b}[0, \infty)}\right): \psi, \psi^{\prime} \in C_{b}^{2}[0, \infty)\right\},
\end{aligned}
$$

$C_{b}^{k}[0, \infty)$

$=\left\{f: f \in C_{b}[0, \infty), k \in \mathbb{N} ;\right.$ such that $\left.\lim _{z \rightarrow \infty} \frac{f(z)}{1+z^{2}}=m_{f}<\infty\right\}$.

For an absolute constant $M>0$, one has

$$
\mathscr{K}_{\psi}(f ; \bar{\delta}) \leq M\left\{\bar{\omega}_{2}(f ; \sqrt{\bar{\delta}})+\min (1, \bar{\delta})\|f\|_{C_{b}[0, \infty)}\right\} .
$$

Let $\bar{\omega}_{2}(f ; \bar{\delta})$ denote the modulus of continuity of order two such that

$$
\bar{\omega}_{2}(f ; \bar{\delta})=\sup _{0<h<\bar{\delta} z[\in[, \infty)} \sup _{0, \infty}|f(z+2 h)-2 f(z+h)+f(z)|
$$

while the classical modulus of continuity is given by

$$
\bar{\omega}(f ; \bar{\delta})=\sup _{0<h<\bar{\delta} z \in[0, \infty)} \sup _{\mid}|f(z+h)-f(z)| .
$$

Theorem 14. For an arbitrary $\varphi \in C_{b}^{2}[0, \infty)$, let an auxiliary operator $\mathcal{S}_{r, \eta}^{*}$ be such that

$$
\begin{aligned}
& \mathscr{K}_{r, \eta}^{*}(\varphi ; z) \\
& \quad=\mathscr{R}_{r, \eta}^{*}(\varphi ; z)+\varphi(z)-\varphi\left\{z+\frac{1}{2 r}\left(2 \frac{L^{\prime}(1)}{L(1)}+4 \eta+1\right)\right\} .
\end{aligned}
$$

Then, for any $\phi \in C_{b}^{2}[0, \infty)$ operators (55), verify the inequality

$$
\begin{aligned}
& \left|\mathscr{K}_{r, \eta}^{*}(\phi ; z)-\phi(y)\right| \\
& \quad \leq\left\{\bar{\delta}_{r, \eta}^{*}(z)++\frac{1}{4 r^{2}}\left(2 \frac{L^{\prime}(1)}{L(1)}+4 \eta+1\right)^{2}\right\}\left\|\psi^{\prime \prime}\right\|,
\end{aligned}
$$

where $\bar{\delta}_{r, \eta}^{*}(z)$ is defined by Theorem 10.

Proof. For any $\phi \in C_{b}^{2}[0, \infty)$, it is easy to verify that $\mathscr{K}_{r, \eta}^{*}$ $\left(\gamma_{0} ; z\right)=1$ and

$$
\begin{aligned}
& \mathscr{K}_{r, \eta}^{*}\left(\gamma_{1} ; z\right) \\
& \quad=\mathscr{R}_{r, \eta}^{*}\left(\gamma_{1} ; z\right)+z-\left\{z+\frac{1}{2 r}\left(2 \frac{L^{\prime}(1)}{\mathrm{L}(1)}+4 \eta+1\right)\right\}=z .
\end{aligned}
$$

We have

$$
\left\|\mathscr{R}_{r, \eta}^{*}(\varphi ; z)\right\| \leq\|\varphi\|,
$$

$$
\begin{aligned}
\left|\mathscr{K}_{r, \eta}^{*}(\varphi ; z)\right| \leq\left|\mathscr{R}_{r, \eta}^{*}(\varphi ; z)\right|+|\varphi(z)|+\mid \varphi \\
\cdot\left\{z+\frac{1}{2 r}\left(2 \frac{L^{\prime}(1)}{\mathrm{L}(1)}+4 \eta+1\right)\right\} \mid \leq 3\|\varphi\| .
\end{aligned}
$$

For any $\phi \in C_{b}^{2}[0, \infty)$, the Taylor series expression gives us

$$
\phi(t)=\phi(z)+(t-z) \phi^{\prime}(z)+\int_{z}^{t}(t-\chi) \phi^{\prime \prime}(\chi) \mathrm{d} \chi .
$$

Therefore, after applying the operators $\mathscr{K}_{r, \eta}^{*}$, on both 
sides we get

$$
\begin{aligned}
\mathscr{K}_{r, \eta}^{*}(\phi ; z)-\phi(z)= & \phi^{\prime}(z) \mathscr{K}_{r, \eta}^{*}\left(\gamma_{1}-z ; z\right)+\mathscr{K}_{r, \eta}^{*} \\
& \cdot\left(\int_{z}^{\gamma_{1}}\left(\gamma_{1}-\chi\right) \phi^{\prime \prime}(\chi) \mathrm{d} \chi ; z\right) \\
= & \mathscr{K}_{r, \eta}^{*}\left(\int_{z}^{t}\left(\gamma_{1}-\chi\right) \phi^{\prime \prime}(\chi) \mathrm{d} \chi ; z\right) \\
= & \mathscr{R}_{r, \eta}^{*}\left(\int_{z}^{\gamma_{1}}\left(\gamma_{1}-\chi\right) \phi^{\prime \prime}(\chi) \mathrm{d} \chi ; z\right) \\
& +\int_{z}^{z}(z-\chi) \phi^{\prime \prime}(\chi) \mathrm{d} \chi ; z \\
& -\int_{z}^{z+(1 / 2 r)\left(2\left(L^{\prime}(1) / \mathrm{L}(1)\right)+4 \eta+1\right)} \\
& \cdot\left(z+\frac{1}{2 r}\left(2 \frac{L^{\prime}(1)}{\mathrm{L}(1)}+4 \eta+1\right)-\chi\right) \phi^{\prime \prime} \\
& \cdot(\chi) \mathrm{d} \chi ;\left|\mathscr{K}_{r, \eta}^{*}(\phi ; z)-\phi(z)\right| \\
\leq & \mathscr{R}_{r, \eta}^{*}\left(\int_{z}^{\gamma_{1}}\left(\gamma_{1}-\chi\right) \phi^{\prime \prime}(\chi) \mathrm{d} \chi ; z\right) \mid \\
& +\mid \int_{z}^{z+(1 / 2 r)\left(2\left(L^{\prime}(1) / \mathrm{L}(1)\right)+4 \eta+1\right)} \\
& \cdot\left(z+\frac{1}{2 r}\left(2 \frac{L^{\prime}(1)}{\mathrm{L}(1)}+4 \eta+1\right)-\chi\right) \phi^{\prime \prime}(\chi) \mathrm{d} \chi \mid .
\end{aligned}
$$

We know the inequality

$$
\begin{gathered}
\quad\left|\int_{z}^{t}(t-\chi) \phi^{\prime \prime}(\chi) \mathrm{d} \chi\right| \leq(t-z)^{2}\left\|\phi^{\prime \prime}\right\|, \\
\mid \int_{z}^{z+(1 / 2 r)\left(2\left(L^{\prime}(1) / \mathrm{L}(1)\right)+4 \eta+1\right)} \\
\quad \times\left(z+\frac{1}{2 r}\left(2 \frac{L^{\prime}(1)}{\mathrm{L}(1)}+4 \eta+1\right)-\chi\right) \phi^{\prime \prime}(\chi) \mathrm{d} \chi \mid \\
\leq\left(\frac{1}{2 r}\left(2 \frac{L^{\prime}(1)}{\mathrm{L}(1)}+4 \eta+1\right)\right)^{2}\left\|\phi^{\prime \prime}\right\| .
\end{gathered}
$$

Thus, we get

$$
\begin{aligned}
& \left|\mathscr{K}_{r, \eta}^{*}(\phi ; z)-\phi(y)\right| \\
& \quad \leq\left\{\mathscr{R}_{r, \eta}^{*}\left(\left(\gamma_{1}-z\right)^{2} ; z\right)+\frac{1}{4 r^{2}}\left(2 \frac{L^{\prime}(1)}{\mathrm{L}(1)}+4 \eta+1\right)^{2}\right\}\left\|\phi^{\prime \prime}\right\| .
\end{aligned}
$$

This gives the complete proof.
Theorem 15. If $\phi \in C_{b}^{2}[0, \infty)$, then for any $f \in C_{b}[0, \infty)$ operators $\mathscr{R}_{r, \eta}^{*}$ by (7) satisfying

$$
\begin{aligned}
& \left|\mathscr{R}_{r, \eta}^{*}(f ; z)-f(z)\right| \\
& \leq \mathscr{M}\left[\bar{\omega}_{2}\left\{f ; \frac{1}{2} \sqrt{\bar{\delta}_{r, \eta}^{*}(z)+\frac{1}{4 r^{2}}\left(2 \frac{L^{\prime}(1)}{L(1)}+4 \eta+1\right)^{2}}\right\}\right. \\
& \quad+\min \left\{1 ; \frac{1}{4}\left(\bar{\delta}_{r, \eta}^{*}(z)+\frac{1}{4 r^{2}}\left(2 \frac{L^{\prime}(1)}{L(1)}+4 \eta+1\right)\right)\right\} \\
& \left.\quad \times\|f\|_{C_{b}[0, \infty)}\right]+\bar{\omega}\left(f ; \frac{1}{2 r}\left(2 \frac{L^{\prime}(1)}{L(1)}+4 \eta+1\right)\right),
\end{aligned}
$$

where $\bar{\delta}_{r, \eta}^{*}(z)$ is defined by Theorem 10.

Proof. We prove Theorem 15 in view of Theorem 14. Therefore, for all $f \in C_{b}[0, \infty)$ and $\phi \in C_{b}^{2}[0, \infty)$, we get

$$
\begin{aligned}
& \left|\mathscr{R}_{r, \eta}^{*}(f ; z)-f(z)\right| \\
& =\left|\mathscr{K}_{r, \eta}^{*}(f ; z)-f(z)+f\left(z+\frac{1}{2 r}\left(2 \frac{L^{\prime}(1)}{\mathrm{L}(1)}+4 \eta+1\right)\right)-f(z)\right| \\
& \leq\left|\mathscr{K}_{r, \eta}^{*}(f-\phi ; z)\right|+\left|\mathscr{K}_{r, \eta}^{*}(\phi ; z)-\phi(z)\right|+|\phi(\mathrm{z})-f(z)| \\
& \quad+\left|f\left(z+\frac{1}{2 r}\left(2 \frac{L^{\prime}(1)}{\mathrm{L}(1)}+4 \eta+1\right)\right)-f(z)\right| \\
& \leq 4\|f-\phi\|+\omega\left(f ;\left|\frac{1}{2 r}\left(2 \frac{L^{\prime}(1)}{\mathrm{L}(1)}+4 \eta+1\right)\right|\right) \\
& \quad+\left\{\bar{\delta}_{r, \eta}^{*}(z)+\frac{1}{4 r^{2}}\left(2 \frac{L^{\prime}(1)}{\mathrm{L}(1)}+4 \eta+1\right)^{2}\right\}\left\|\psi^{\prime \prime}\right\| .
\end{aligned}
$$

If we take infimum for all $\phi \in C_{b}^{2}[0, \infty)$, then in view of (50) it is easy to conclude that

$$
\begin{aligned}
\left|\mathscr{R}_{r, \eta}^{*}(f ; z)-f(z)\right| & \\
\leq & 4 K_{2}\left\{f ; \frac{1}{4}\left(\bar{\delta}_{r, \eta}^{*}(z)+\frac{1}{4 r^{2}}\left(2 \frac{L^{\prime}(1)}{\mathrm{L}(1)}+4 \eta+1\right)\right)\right\} \\
& +\bar{\omega}\left(f ; \frac{1}{2 r}\left(2 \frac{L^{\prime}(1)}{\mathrm{L}(1)}+4 \eta+1\right)\right) \\
\leq & \mathscr{M}\left[\bar{\omega}_{2}\left\{f ; \frac{1}{2} \sqrt{\bar{\delta}_{r, \eta}^{*}(z)+\frac{1}{4 r^{2}}\left(2 \frac{L^{\prime}(1)}{\mathrm{L}(1)}+4 \eta+1\right)^{2}}\right\}\right. \\
& +\min \left\{1 ; \frac{1}{4}\left(\bar{\delta}_{r, \eta}^{*}(z)+\frac{1}{4 r^{2}}\left(2 \frac{L^{\prime}(1)}{\mathrm{L}(1)}+4 \eta+1\right)\right)\right\} \\
& \left.\times\|f\|_{C_{b}[0, \infty)}\right]+\bar{\omega}\left(f ; \frac{1}{2 r}\left(2 \frac{L^{\prime}(1)}{\mathrm{L}(1)}+4 \eta+1\right)\right) .
\end{aligned}
$$

The proof is completed here. 
Now, we give the local direct estimate for the operators $\mathscr{R}_{r, \eta}^{*}$ defined by (7) via the well-known Lipschitz-type maximal function involving the parameters $\mu, v>0$ and number $\lambda \in(0,1]$. Thus, from [26], we recall that

$$
\begin{aligned}
\operatorname{Lip}_{\mathscr{L}}^{\lambda}=\{f & \left\{C_{b}[0, \infty):|f(t)-f(z)|\right. \\
& \left.\leq \mathscr{L} \frac{|t-z|^{\lambda}}{\left(\mu z^{2}+v z+t\right)^{\lambda / 2}} ; z, t \in[0, \infty)\right\},
\end{aligned}
$$

where $\mathscr{L}$ is a positive constant.

Theorem 16. For any $f \in \operatorname{Lip}_{\mathscr{L}}^{\lambda}$ satisfied by (70), operators $\mathscr{R}_{r, \eta}^{*}$ hold the inequality

$$
\left|\mathscr{R}_{r, \eta}^{*}(f ; z)-f(z)\right| \leq \mathscr{L}\left(\frac{\bar{\delta}_{r, \eta}^{*}(z)}{\left(\mu z^{2}+v z\right)}\right)^{\lambda / 2}
$$

where $\bar{\delta}_{r, \eta}^{*}(z)$ is obtained by Theorem 10.

Proof. Let $f \in \operatorname{Lip}_{\mathscr{L}}^{\lambda}$ for $0<\lambda \leq 1$; then, first we verify the results are true when $\lambda=1$. For any $\mu, v \geq 0$, it is easy to use the result $\left(\mu z^{2}+\nu z+t\right)^{-1 / 2} \leq\left(\mu z^{2}+v z\right)^{-1 / 2}$ and then we apply the Cauchy-Schwarz inequality. Thus, we can write

$$
\begin{aligned}
\left|\mathscr{R}_{r, \eta}^{*}(f ; z)-f(z)\right| & \leq\left|\mathscr{R}_{r, \eta}^{*}(|f(t)-f(z)| ; z)\right|+f(z)|(1 ; z)-1| \\
& \leq \mathscr{R}_{r, \eta}^{*}\left(\frac{|t-z|}{\left(\mu z^{2}+v z+t\right)^{1 / 2}} ; z\right) \\
& \leq \mathscr{L}\left(\mu z^{2}+v z\right)^{-1 / 2} \mathscr{R}_{r, \eta}^{*}(|t-z| ; z) \\
& \leq \mathscr{L}\left(\mu z^{2}+v z\right)^{-1 / 2} \mathscr{R}_{r, \eta}^{*}\left(\gamma_{1}-z\right)^{2} ;\left.z\right|^{1 / 2} .
\end{aligned}
$$

From these conclusions, we get that the statement holds for $\lambda=1$. Now, we check if the statement is valid if $0<\lambda<$ 1. For this reason, we use monotonicity property to $\mathscr{R}_{r, \eta}^{*}$ and apply the well-known Hölder inequality

$$
\begin{aligned}
\left|\mathscr{R}_{r, \eta}^{*}(f ; z)-f(z)\right| \leq & \mathscr{R}_{r, \eta}^{*}(|f(t)-f(z)| ; z) \\
\leq & \left(\mathscr{R}_{r, \eta}^{*}\left(|f(t)-f(z)|^{2 / \lambda} ; z\right)\right)^{\lambda / 2} \\
& \cdot\left(\mathscr{R}_{r, \eta}^{*}\left(\gamma_{0} ; z\right)\right)^{(2-\lambda) / 2} \\
\leq & \mathscr{L}\left\{\frac{\mathscr{R}_{r, \eta}^{*}\left(\left(\gamma_{1}-z\right)^{2} ; z\right)}{t+\mu z^{2}+v z}\right\}^{\lambda / 2}
\end{aligned}
$$

$$
\begin{aligned}
& \leq \mathscr{L}\left(\mu z^{2}+v z\right)^{-\lambda / 2}\left\{\mathscr{R}_{r, \eta}^{*}\left(\left(\gamma_{1}-z\right)^{2} ; z\right)\right\}^{\lambda / 2} \\
& \leq \mathscr{L}\left(\mu z^{2}+v z\right)^{-\lambda / 2}\left(\mathscr{R}_{r, \eta}^{*}\left(\gamma_{1}-z\right)^{2} ; z\right)^{\lambda / 2} \\
& =\mathscr{L}\left(\frac{\bar{\delta}_{r, \eta}^{*}(z)}{\left(\mu z^{2}+v z\right)}\right)^{\lambda / 2},
\end{aligned}
$$

which completes the proof.

Here, we obtain the other local approximation results of $\mathscr{R}_{r, \eta}^{*}$ in Lipschitz spaces. For all Lipschitz maximal function $f \in C_{b}[0, \infty), 0<\lambda \leq 1$ and $t, z \in[0, \infty)$, from [27] we recall that

$$
\bar{\omega}_{\lambda}(f ; z)=\sup _{t \neq z, t \in[0, \infty)} \frac{|f(t)-f(z)|}{|t-z|^{\lambda}}
$$

Theorem 17. Let $f \in C_{b}[0, \infty)$, then for all $z \in[0, \infty)$,

$$
\left|\mathscr{R}_{r, \eta}^{*}(f ; z)-f(z)\right| \leq\left(\bar{\delta}_{r, \eta}^{*}(z)\right)^{\lambda / 2} \bar{\omega}_{\lambda}(f ; z),
$$

where $\bar{\omega}_{\lambda}(f ; z)$ is obtained in and $\bar{\delta}_{r, \eta}^{*}(z)$ is defined by Theorem 10.

Proof. From the well-known Hölder inequality, we get

$$
\begin{aligned}
\left|\mathscr{R}_{r, \eta}^{*}(f ; z)-\mathrm{f}(z)\right| \leq & \mathscr{R}_{r, \eta}^{*}(|f(t)-f(z)| ; z) \\
\leq & \bar{\omega}_{\lambda}(f ; z) \mid \mathscr{R}_{r, \eta}^{*}\left(|t-z|^{\lambda} ; z\right) \\
\leq & \bar{\omega}_{\lambda}(f ; z)\left(\mathscr{R}_{r, \eta}^{*}\left(\gamma_{0} ; z\right)\right)^{(2-\lambda) / 2} \\
& \cdot\left(\mathscr{R}_{r, \eta}^{*}\left(|t-z|^{2} ; z\right)\right)^{\lambda / 2} \\
= & \bar{\omega}_{\lambda}(f ; z)\left(\mathscr{R}_{r, \eta}^{*}\left(\left(\gamma_{1}-z\right)^{2} ; z\right)\right)^{\lambda / 2} .
\end{aligned}
$$

Thus, we get the proof.

\section{Voronovskaja-Type Approximation Theorems}

In this section, we establish a quantitative Voronovskaja-type theorem for the operators $\mathscr{R}_{r, \eta}^{*}(f ; z)$. 
Theorem 18. Let $f \in C_{b}[0, \infty)$, then for each $z \in[0, \infty)$

$$
\begin{aligned}
\lim _{r \rightarrow \infty} r\left\{\mathscr{R}_{r, \eta}^{*}(\psi ; z)-\psi(z)\right\}= & \left(2 \frac{L^{\prime}(1)}{L(1)}+4 \eta+1\right) \frac{\psi^{\prime}(z)}{2} \\
& +\frac{z \psi^{\prime \prime}(z)}{2},
\end{aligned}
$$

where $\psi^{\prime}(z), \psi^{\prime \prime}(z) \in C_{b}[0, \infty)$.

Proof. From the expression of Taylor's expansion of function $\psi(z)$ in $C_{b}[0, \infty)$, we write

$\psi(t)=\psi(z)+(t-z) \psi^{\prime}(z)+\frac{1}{2}(t-z)^{2} \psi^{\prime \prime}(z)+(t-z)^{2} Q_{z}(t)$

where $Q_{z}(t)$ is the remainder term and $Q_{z} \in[0, \infty)$ with $Q_{z}(t) \rightarrow 0$ as $t \rightarrow z$. On applying the operators $\mathscr{R}_{r, \eta}^{*}(\cdot ; z)$ to (74), then use the Cauchy-Schwarz inequality. Thus, we get

$$
\begin{aligned}
\mathscr{R}_{r, \eta}^{*}(\psi ; z)-\psi(z)= & \psi^{\prime}(z) \mathscr{R}_{r, \eta}^{*}\left(\gamma_{1}-z ; z\right)+\frac{\psi^{\prime \prime}(z)}{2} \mathscr{R}_{r, \eta}^{*} \\
& \cdot\left(\left(\gamma_{1}-z\right)^{2} ; z\right)+\mathscr{R}_{r, \eta}^{*}\left(\left(\gamma_{1}-z\right)^{2} Q_{z}\left(\gamma_{1}\right) ; z\right) \\
\leq & \psi^{\prime}(\mathrm{z}) \mathscr{R}_{r, \eta}^{*}\left(\gamma_{1}-z ; z\right)+\frac{\psi^{\prime \prime}(z)}{2} \mathscr{R}_{r, \eta}^{*} \\
& \cdot\left(\left(\gamma_{1}-z\right)^{2} ; z\right) \\
& +\sqrt{\mathscr{R}_{r, \eta}^{*}\left(\left(\gamma_{1}-z\right)^{4} ; z\right)} \sqrt{\mathscr{R}_{r, \eta}^{*}\left(Q_{z}^{2}\left(\gamma_{1}\right) ; z\right) .}
\end{aligned}
$$

Since we have $\lim _{r \rightarrow \infty} \mathscr{R}_{r, \eta}^{*}\left(Q_{z}^{2}\left(\gamma_{1}\right) ; z\right)=0$, therefore

$$
\lim _{r \rightarrow \infty} r\left\{\mathscr{R}_{r, \eta}^{*}\left(\left(\gamma_{1}-z\right)^{2} Q_{z}\left(\gamma_{1}\right) ; z\right)\right\}=0
$$

Thus, we have

$$
\begin{aligned}
& \lim _{r \rightarrow \infty} r\left\{\mathscr{R}_{r, \eta}^{*}(\psi ; z)-\psi(z)\right\} \\
& =\lim _{r \rightarrow \infty} r\left\{\mathscr{R}_{r, \eta}^{*}\left(\gamma_{1}-z ; z\right) \psi^{\prime}(z)+\frac{\psi^{\prime \prime}(z)}{2} \mathscr{R}_{r, \eta}^{*}\left(\left(\gamma_{1}-z\right)^{2} ; z\right)\right. \\
& \left.\quad+\mathscr{R}_{r, \eta}^{*}\left(\left(\gamma_{1}-z\right)^{2} Q_{z}\left(\gamma_{1}\right) ; z\right)\right\},
\end{aligned}
$$

which completes the proof.

As a consequence of Theorem 18, we immediately get the corollary.
Corollary 19. For any $\psi \in C[0, \infty)$, we have

$$
\begin{aligned}
\lim _{r \rightarrow \infty} r & {\left[\mathscr{R}_{r, \eta}^{*}(\psi ; z)-\psi(z)-\frac{1}{2 r}\left(2 \frac{L^{\prime}(1)}{L(1)}+4 \eta+1\right) \psi^{\prime}(z)\right.} \\
& \left.-\frac{\bar{\delta}_{r, \eta}^{*}(z)}{2} \psi^{\prime \prime}(z)\right]=0 .
\end{aligned}
$$

\section{Conclusion}

Motivated by article [5], we have introduced a Kantorovich generalization of the Szász-Mirakjan operators by Dunkl analogue involving the Appell polynomials. These types of generalizations enable to give the generalized results rather than the earlier study demonstrations by $[3,5,7]$. Lastly, we have also discussed the Voronovskaja-type approximation theorems of these new operators.

\section{Data Availability}

No data were used to support this study.

\section{Conflicts of Interest}

The authors are very grateful and declare that they have no competing interest.

\section{Authors' Contributions}

All authors read and agreed to the contents of this research article.

\section{References}

[1] O. Szász, "Generalization of Bernstein's polynomials to the infinite interval," Journal of Research of the National Bureau of Standards, vol. 45, no. 3, pp. 239-245, 1950.

[2] S. N. Bernstein, "Démonstration du théorème de Weierstrass fondée sur le calcul des probabilités," Communications de la Société mathématique de Kharkow, vol. 13, no. 1, pp. 1-2, 1912.

[3] S. Sucu, "Dunkl analogue of Szasz operators," Applied Mathematics and Computation, vol. 244, pp. 42-48, 2014.

[4] P. Appell, "Sur une classe de polynômes," Annales scientifiques de l'École normale supérieure, vol. 9, pp. 119-144, 1880.

[5] M. Nasiruzzaman and A. F. Aljohani, "Approximation by Szász-Jakimovski-Leviatan type operators via aid of Appell polynomials," Journal of function spaces, Article ID 9657489, 11 pages, 2020.

[6] A. Alotaibi, M. Mursaleen, 1 Operator Theory and Applications Research Group, Department of Mathematics, Faculty of Science, King Abdulaziz University, Jeddah, Saudi Arabia, 2 Department of Medical Research, China Medical University Hospital, China Medical University (Taiwan), Taichung, Taiwan, 3 Department of Mathematics, Aligarh Muslim University, Aligarh 202002, India, and 4 Department of Computer Science and Information Engineering, Asia University, Taichung, Taiwan, "Approximation of Jakimovski-Leviatan-Beta type integral operators via q-calculus," AIMS Mathematics, vol. 5, no. 4, pp. 3019-3034, 2020. 
[7] G. Içöz and B. Çekim, "Stancu-type generalization of Dunkl analogue of Szász-Kantorovich operators," Mathematical Methods in the Applied Sciences, vol. 39, no. 7, pp. 18031810, 2016.

[8] A. Jakimovski and D. Leviatan, "Generalized Szász operators for the approximation in the infinite interval," Mathematica (Cluj), vol. 11, no. 34, pp. 97-103, 1969.

[9] S. A. Mohiuddine, T. Acar, and A. Alotaibi, "Construction of a new family of Bernstein-Kantorovich operators," Mathematical Methods in the Applied Sciences, vol. 40, no. 18, pp. 77497759, 2017.

[10] S. A. Mohiuddine, B. Hazarika, and M. A. Alghamdi, "Ideal relatively uniform convergence with Korovkin and Voronovskaya types approximation theorems," Filomat, vol. 33, no. 14, pp. 4549-4560, 2019.

[11] M. Mursaleen, K. J. Ansari, and M. Nasiruzzaman, “Approximation by q-analogue of Jakimovski-Leviatan operators involving q-Appell polynomials," Iranian Journal of Science and Technology, Transactions A: Science, vol. 41, no. 4, pp. 891-900, 2017.

[12] M. Mursaleen and M. Nasiruzzaman, "Approximation of modified Jakimovski-Leviatan-Beta type operators," Constructive Mathematical Analysis, vol. 1, no. 2, pp. 88-98, 2018.

[13] M. Mursaleen, M. Nasiruzzaman, and A. Alotaibi, "On modified Dunkl generalization of Szász operators via q-calculus," Journal of Inequalities and Applications, vol. 2017, no. 1, 2017.

[14] A. Kilicman, M. A. Mursaleen, and A. A. H. Al-Abied, "Stancu type Baskakov-Durrmeyer operators and approximation properties," Mathematics, vol. 8, no. 7, 2020.

[15] S. A. Mohiuddine and F. Özger, "Approximation of functions by Stancu variant of Bernstein-Kantorovich operators based on shape parameter $\alpha$," Revista de la Real Academia de Ciencias Exactas, Físicas y Naturales. Serie A. Matemáticas, vol. 114, no. 2, 2020.

[16] M. Mursaleen, F. Khan, and A. Khan, "Approximation properties for King's type modifiedq-Bernstein-Kantorovich operators," Mathematical Methods in the Applied Sciences, vol. 38, no. 18, pp. 5242-5252, 2015.

[17] M. Mursaleen and T. Khan, "On approximation by Stancu type Jakimovski-Leviatan-Durrmeyer operators," Azerbaijan Journal of Mathematics, vol. 7, no. 1, pp. 16-26, 2017.

[18] M. Mursaleen, A. A. H. Al-Abied, and M. A. Salman, "Chlodowsky type $(\lambda ; \mathrm{q})$-Bernstein-Stancu operators," Azerbaijan Journal of Mathematics, vol. 10, no. 1, pp. 75-101, 2020.

[19] F. Özger, "Weighted statistical approximation properties of univariate and bivariate $\lambda$-Kantorovich operators," Filomat, vol. 33, no. 11, pp. 3473-3486, 2019.

[20] H. M. Srivastava, F. Özger, and S. A. Mohiuddine, "Construction of Stancu-type Bernstein operators based on Bézier bases with shape parameter $\lambda$," Symmetry, vol. 11, no. 3, 2019.

[21] A. D. Gadziev, "Theorems of the type of P.P," in Korovkin's theorems, vol. 20 of Matematicheskie Zametki, no. 5, pp. 781-786, 1976.

[22] P. P. Korovkin, Linear Operators and Approximation Theory, Hindustan Publishing Corporation, Delhi, 1960.

[23] A. D. Gadjiev, "The convergence problem for a sequence of positive linear operators on bounded sets and theorems analogous to that of P. P. Korovkin," Doklady Akademii Nauk SSSR, vol. 218, no. 5, pp. 1001-1004, 1974.

[24] O. Shisha and B. Mond, "The degree of convergence of sequences of linear positive operators," Proceedings of the
National Academy of Sciences of the United States of America, vol. 60, no. 4, pp. 1196-1200, 1968.

[25] Ç. Atakut and N. Ispir, "Approximation by modified SzászMirakjan operators on weighted spaces," Proceedings of the Indian Academy of Sciences-Mathematical Sciences, vol. 112, pp. 571-578, 2002.

[26] M. A. Ozarslan and H. Aktug lu, "Local approximation for certain King type operators," Filomat, vol. 27, pp. 173-181, 2013.

[27] B. Lenze, "On Lipschitz type maximal functions and their smoothness spaces," Indagationes Mathematicae (Proceedings), vol. 91, no. 1, pp. 53-63, 1988. 